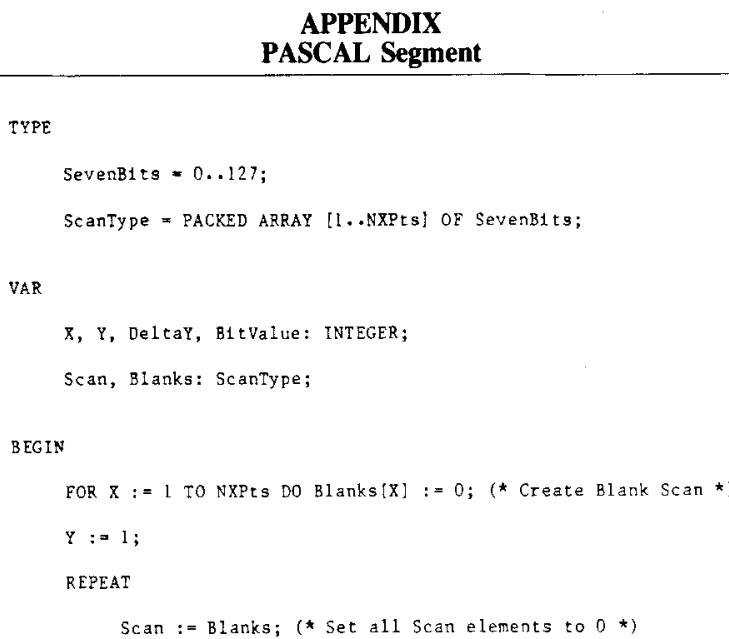

APPENDIX

PASCAL Segment

TYPE

SevenB1ts $=0 . .127$

Scantype = PACKED ARRAY [?,.NXPts] OE SevenB1tg;

VAR

$X, Y$, DeltaY, B1tValue: INTEGER

Scan, Blanks: ScanType;

BEGIN

FOR $X:=1$ TO NXPts DO Blanks $[X]:=0 ;$ (* Create Blank Scan *)

Y : : 1 ;

REPEAT

Scan : = Blanks; (* Set all Scan elements to 0 *)

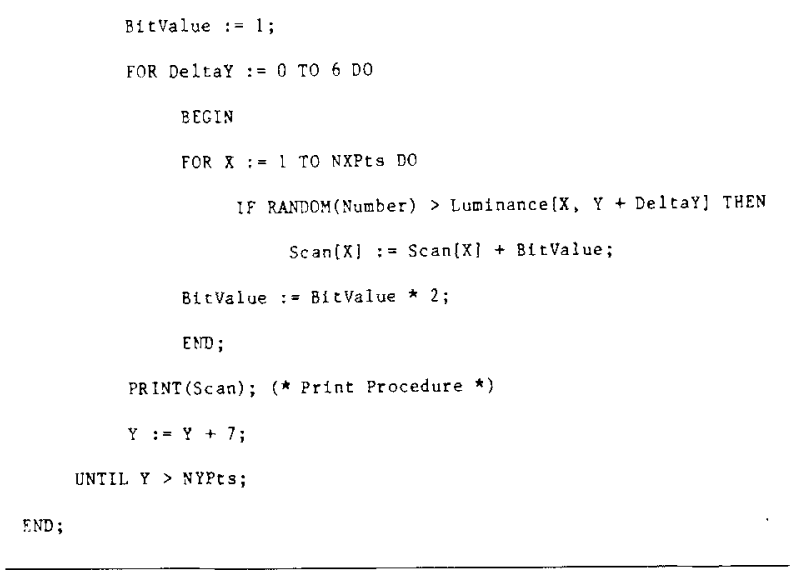

(Manuscript accepted for publication December 14, 1984.)

\title{
Announcement
}

\section{Artificial Intelligence Consortium Founded}

Eight universities have entered into a 5-year, $\$ 8,236,000$ contract with the U.S. Air Force's Rome Air Development Center (RADC) to study artificial intelligence - the application of computer systems to problems that could previously be solved only by human intelligence.

"The project is aimed at establishing a major research force in artificial intelligence in the Northeast," said Fred Diamond, chief scientist at the RADC at Griffiss Air Force Base in Rome, NY.

Members of the new Artificial Intelligence Consortium (AIC) are: Syracuse University, University of Rochester, Rochester Institute of Technology, State University of New York at Buffalo, Rensselaer Polytechnic Institute, Clarkson University, Colgate University, and University of Massachusetts. The Air Force Institute of Technology at Wright-Patterson Air Force Base in Ohio will participate in the program on a nonfunded basis. This unique cooperative effort is cosponsored by the Rome Air Development Center and the Air Force Office of Scientific Research, both organizations of the Air Force Systems Command.

Preparation of the funding proposal was organized and directed by Virgil Eveleigh, professor of electrical and computer engineering at Syracuse University, with major input by Syracuse University professors P. Bruce Berra, also in electrical and computer engineering, and Kenneth Bowen of the School of Computer and Information Science. Syracuse University will be the prime contractor with the Air Force and will negotiate subcontracting agreements with the other member institutions.

Although Syracuse University is the focal point for the AI Consortium, each member institution will contribute researchon an equal basis-in its respective area of expertise. Roles may change or expand as the project matures.

Initial research interests of the member institutions are:

-Syracuse University-logic programming and the development of special-purpose computer hardware for typical AI applications;

-University of Rochester-problem solving, and temporal relationships and reasonings (which involves ordering the actions of devices monitored by a computer);

- Rochester Institute of Technology-speech-understanding systems, including microphone speech processing;

-SUNY Buffalo-versatile expert maintenance systems;

-Rennselaer Polytechnic Institute-image-understanding systems, aerial and satellite imagery (both electronic and photographic);

-Clarkson University-distributed problem solving;

- Colgate University-natural language processing and plan recognition research;

-University of Massachusetts-intelligent user interfaces, natural language generation and understanding, and distributed artificial intelligence (which involves the coordination of expert systems).

The Artificial Intelligence Consortium has four main goals: to improve the Air Force's technical AI abilities; to improve the training of Air Force technicians and scientists and expand the general base of AI expertise; to stimulate business involvement in AI research and development; and to establish a productive, cooperative relationship among institutions distinguished by their research in this field.

Scientists and engineers believe AI techniques will enable them to develop advanced computer systems that not only can process vast amounts of information, but also can interpret, predict, analyze, and explain such data.

For further information, contact: Anthony D'Angelo, Syracuse University, 105 Administration Building, Syracuse, NY 13210. 